\title{
Integration of photoacoustic imaging and high-intensity focused ultrasound
}

\author{
Huizhong Cui \\ Jacob Staley \\ Xinmai Yang \\ University of Kansas \\ KU Bioengineering Research Center \\ Department of Mechanical Engineering \\ 1530 West 15th Street \\ Lawrence, Kansas 66045
}

\begin{abstract}
We have developed an integrated photoacoustic imaging (PAI) and high-intensity focused ultrasound (HIFU) system for solid tumor treatments. A single-element, spherically focused ultrasonic transducer, with a central frequency of $5 \mathrm{MHz}$, was used to induce HIFU lesions in soft tissue. The same ultrasonic transducer was also used as a detector during PAI to guide HIFU ablation. The use of same transducer for PAI and HIFU can reduce the requirement on acoustic windows during the imaging-guided therapy, as well as ensuring the correct alignment between the therapeutic beam and the planned treatment volume. During an experiment, targeted soft tissue was first imaged by PAI. The resulted image was used to plan the subsequent HIFU ablation. After the HIFU ablation, targeted soft tissue was imaged again by PAI to evaluate the effectiveness of treatments. Good contrast was obtained between photoacoustic images before and after HIFU ablation. In conclusion, our results demonstrated that PAI technology may potentially be integrated with HIFU ablation for imageguided therapy. (- 2010 Society of Photo-Optical Instrumentation Engineers. [DOI: 10.1117/1.3365948]
\end{abstract}

Keywords: high-intensity focused ultrasound (HIFU); photoacoustic imaging (PAI); image-guided therapy.

Paper 09319SSRR received Jul. 31, 2009; revised manuscript received Jan. 4, 2010; accepted for publication Jan. 15, 2010; published online Mar. 23, 2010.

\section{Introduction}

High-intensity focused ultrasound (HIFU) has been used as an effective noninvasive method to treat solid tumors deep in the body. During HIFU treatment, high-intensity ultrasound is delivered to the focal region of the HIFU transducer and subsequently absorbed by the soft tissue in the focal region. The absorption of HIFU energy induces a rapid temperature rise, which results in tissue coagulation, and finally leads to the irreversible tumor cell death and severe damage to tumor blood vessels in the treated region. ${ }^{1,2}$ The soft tissue outside the focal region will remain undamaged because the ultrasound intensity is relatively low in those regions.

To improve the effectiveness of HIFU treatment, we need to locate and monitor the treated tissue so that the guidance and feedback on the treatment can be provided. ${ }^{3}$ Current imaging modalities, such as magnetic resonance imaging (MRI) and ultrasound imaging, have been used to visualize the treatment process and monitor the immediate thermal effects. ${ }^{4-8}$ However, MRI makes the treatment cumbersome and expensive. Although ultrasound imaging is inexpensive and can potentially perform with the same HIFU transducer in real time, it has relatively low imaging contrast, sensitivity, and specificity for noninvasive detection.

Photoacoustic imaging (PAI), also called optoacoustic imaging or thermoacoustic imaging, has been developed as a

Address all correspondence to: Xinmai Yang, KU Bioengineering Research Center, Department of Mechanical Engineering, University of Kansas, 1530 West 15th Street, Lawrence, Kansas 66045. Tel: 785-864-1753; Fax: 785-864-1742; E-mail: xmyang@ku.edu novel promising imaging technique for early cancer detection. $^{9-14}$ PAI is based on the generation of photoacoustic waves by safely depositing short-pulsed optical energy into tissue. Each laser pulse causes a rapid temperature rise on the order of $10 \mathrm{mdeg}$. The ultrasonic waves generated due to thermoelastic expansion can be detected by either a singleelement ultrasonic transducer or an array of ultrasonic transducers and then used to reconstruct an image. ${ }^{14}$ PAI technology is designed to overcome the poor spatial resolution of purely optical imaging yet to retain the high optical contrast in the deep region. PAI has demonstrated the capability of providing images with optical contrast and ultrasound resolution in regions up to $5 \mathrm{~cm}$ deep in soft tissue. ${ }^{15}$

PAI has demonstrated to be capable of monitoring thermal lesions generated by HIFU or other means. ${ }^{16,17}$ A study for PAI guidance of HIFU has also been proposed with the application of a time-reversal technique. ${ }^{18}$ Currently, PAI and HIFU are performed by two separate systems. When PAI and HIFU are performed separately by two systems, a tissueimaging window for PAI, which utilizes both light and ultrasound, and a therapeutic window for HIFU to deliver ultrasound energy will be required. In clinical practice, two tissue windows are sometimes difficult to identify. In addition, when two separate systems are used, the location of both systems needs to be known, precisely, so that the treatment will be performed on the lesion, which is identified on the image. To align the treatment area with the imaging area could be a challenge and may result in the ablation of normal tissue.

$1083-3668 / 2010 / 15(2) / 021312 / 4 / \$ 25.00$ @ $2010 \mathrm{SPIE}$ 


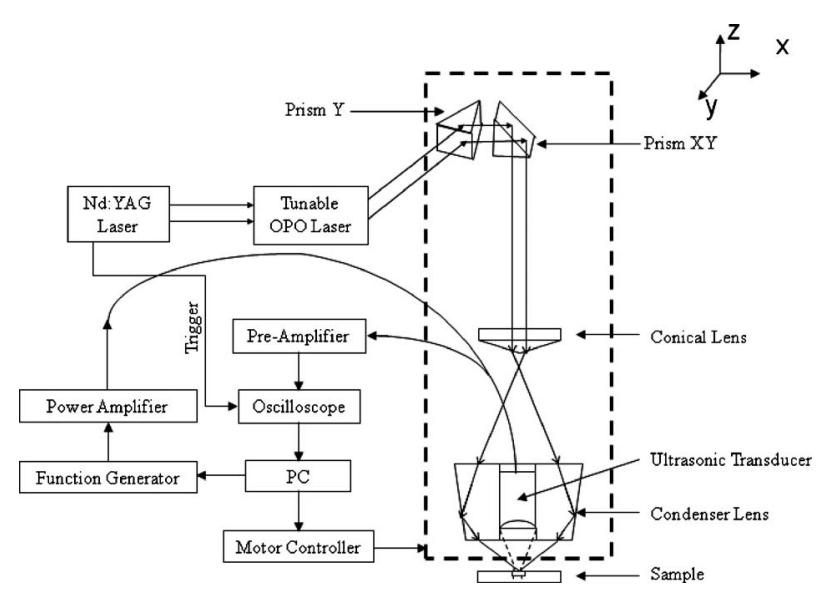

Fig. 1 Schematic of the integrated PAI and HIFU system.

One way to improve PAI-guided HIFU is to use an integrated system for PAI and HIFU. The combination of PAI and HIFU is technically possible. In PAI technology, both laser and ultrasound energy are involved in the imaging process. The ultrasonic transducer's sole purpose within the modality is to act as a receiver for the acoustic signals. If the ultrasonic transducer is used as a transmitter to deliver high ultrasound energy to soft tissue, the PAI system potentially has therapeutic applications as a HIFU system.

HIFU can treat soft tissues up to $10 \mathrm{~cm}$, whereas the deepreflection-mode $\mathrm{PAI}$, when a $5-\mathrm{MHz}$ ultrasonic transducer is used, can provide penetration depth up to $\sim 3.8 \mathrm{~cm}$ without using any contrast agents. ${ }^{19}$ With the aid of PAI contrast agents, the deep-reflection-mode PAI should be able to image even deeper. Therefore, a reflection-mode PAI-guided HIFU may be used to treat breast tumor, prostate tumor, tumors in the lymphatic system, etc.

In this paper, we investigated the feasibility of using an integrated PAI and HIFU system to noninvasively detect the location of the target tissue sample and then plan and perform the HIFU ablation on the target tissue sample. The biggest advantage of a combined reflection-mode PAI and HIFU with one transducer lies on the fact that only one tissue-imaging/ therapy window is required with this technique, and therefore, it will be convenient to use in future clinical practice. Furthermore, with the combined system, the treatment can be performed precisely on the area identified through the obtained image and, therefore, the treatment location can be very precise. This advantage may be of more interest when treating small lesions, which may be identified in early cancer detection.

In the combined PAI/HIFU system, we utilize a reflectionmode PAI system (also called PAM) ${ }^{19,20}$ with a standard 5-MHz HIFU transducer as our photoacoustic signal detector. The HIFU transducer also delivers high-intensity ultrasound energy to soft tissue during tissue ablations. Our results show that the combined PAI/HIFU is capable of performing imaging and therapy.

\section{Materials and Methods}

Figure 1 shows the schematic of the integrated PAI and HIFU system. The system can be operated at PAI or HIFU mode.
When the system is operated at PAI mode, a tunable OPO laser (Surelite OPO PLUS; Continuum, Santa Clara, California), pumped by a Q-switched Nd:YAG laser (Surelite III; Continuum) is used to generate laser light. The system operates at a $680-\mathrm{nm}$ wavelength with a $10-\mathrm{Hz}$ pulse repetition rate for excitation of the tissue. The produced laser light is directed to a conical lens via a group of prisms, which enable higher optical energy delivery ${ }^{19}$ than the optical fibers-based PAI systems. ${ }^{20}$ The laser light passing through the conical lens forms a ring-shaped illumination. The ring-shaped light is transmitted to an optical condenser and refocused inside the tissue sample. At the tissue surface, the ring has a diameter of $\sim 5 \mathrm{~mm}$, which has the advantage of reducing the generation of surface photoacoustic signals and allowing for the improvement in the detection of photoacoustic signals from deep regions. ${ }^{19,20}$ The subsequently generated photoacoustic signals are detected by a $5-\mathrm{MHz}$ focused ultrasonic transducer (SU108-013, Sonic Concepts, Bothell, Washington), which has a focal length of $35 \mathrm{~mm}$ and a diameter of $33 \mathrm{~mm}$. The transducer delivers the recorded acoustic signals to a preamplifier (5072PR, Olympus-NDT, Waltham, Massachusetts). After signal amplification, the detected photoacoustic signals are collected by a PC through a digital oscilloscope (Tektronix DPO 3034, Beaverton, Oregon). The transducer is mounted in the middle of the condenser lens and driven by an $X Y Z$-linear translation stage for raster scanning on the tissue sample. Two prisms are used to enable the beam folding for the 2-D scan. Prism $Y$ can only move on the $Y$ direction, whereas prism $X Y$ can move on both $X$ and $Y$ direction. This design has been presented by Song and Wang ${ }^{19}$ for deep PAI, and proved to generate good-resolution photoacoustic images in deep regions inside soft tissue.

When the system is operated at HIFU mode, the 5-MHz focused ultrasonic transducer is used as a transmitter. At each scanning position, continuous ultrasonic waves, which are generated by a function generator (HP33250A; Agilent Technologies, Santa Clara, California) and amplified by a $50-\mathrm{dB}$ radio frequency (RF) amplifier (350L; ENI Technology, Inc., Rochester, New York), are sent to the transducer. The HIFU field is then induced step by step in the tissue sample while the transducer is driven by the $X Y Z$-linear translation stage.

In order to quantify the actual resolution of our PAI system with a HIFU transducer as the receiver, a well-controlled sample (human hair, $\sim 50 \mu \mathrm{m}$ diam) in water was imaged. Figure 2 shows the cross section of the human hair on a B-mode scan image. We can achieve a lateral resolution of $\sim 270 \mu \mathrm{m}$ and an axial resolution of $\sim 200 \mu \mathrm{m}$.

To test the ability of the system in the detection of optical contrast change before and after HIFU, we performed an in vitro test in a chicken liver. A slice (2-mm thick) of chicken liver was immersed in water and first imaged by PAI, and then HIFU was used to insonificate a portion of the liver. After HIFU sonication, PAI was used to image the liver slice again.

To further investigate the tumor detections by PAI and localized ablation by HIFU, we embedded a thin slice of swine liver tissue (2-mm thick) between two fresh chicken breast tissues slabs. The thicknesses of the two chicken breast slabs were different. The upper slab was $\sim 1$-mm thick, while the lower slab was much larger, $\sim 10 \mathrm{~mm}$ thick, in order to reduce the interference from signals generated at the bottom of 


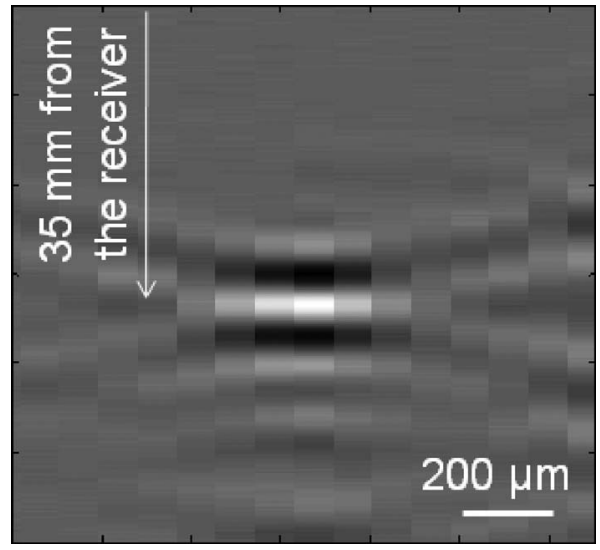

Fig. 2 Cross section of a human hair in water.

the sample. The samples were immersed in water, and air bubbles were carefully removed before PAI. Because the optical absorption in the swine liver tissue is much higher than that in the chicken breast tissue, ${ }^{21}$ good image contrast should be achieved between them. During the experiments, PAI was first used to detect the location of the swine liver and then, HIFU ablation was performed based on the exact position of the swine liver sample identified on the photoacoustic image. After HIFU ablation, PAI was performed again to detect the change on the generated photoacoustic signals.

For the photoacoustic image formation, we took maximum-amplitude-projected (MAP) image. On each MAP image, the maximum value of the time-resolved photoacoustic signal detected at each scan position was plotted.

\section{Results}

Figure 3(a) shows the photoacoustic image of the chicken liver after a portion of the liver went through the HIFU ablation, whereas Fig. 3(b) shows a photograph of the chicken liver. Compared to the untreated region, the HIFU-treated region shows a great enhancement in the amplitude of photoacoustic signals. On the image, the averaged contrast ratio between the treated and untreated region is $\sim 3.6$, with a

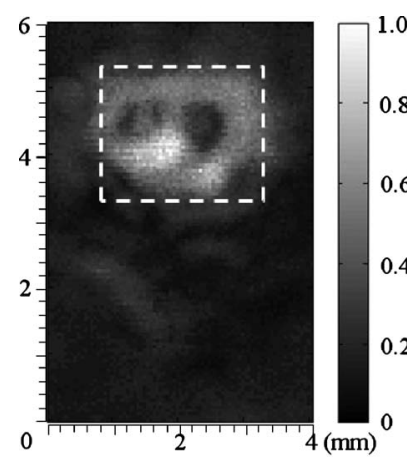

(a)

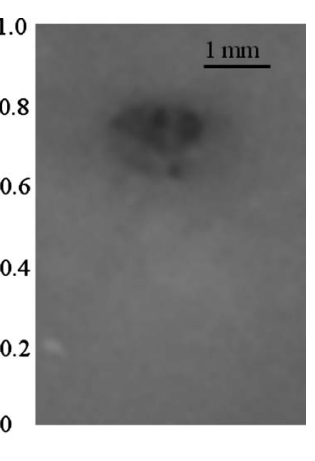

(b)
Fig. 3 (a) Photoacoustic image of a chicken liver after HIFU ablation. The ultrasound intensity at the focal zone is $10^{4} \mathrm{~W} / \mathrm{cm}^{2}$, the ablation duration is $1 \mathrm{~s}$ at each scanning position. (b) Photograph of the chicken liver after HIFU ablation. The HIFU targeted area is outlined on (a). a)
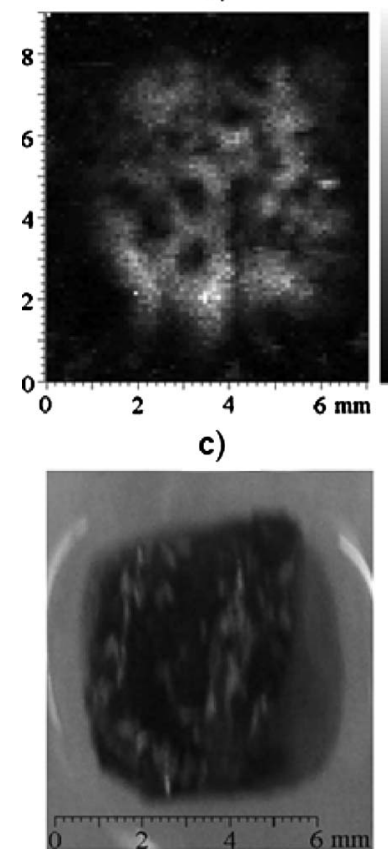

b)

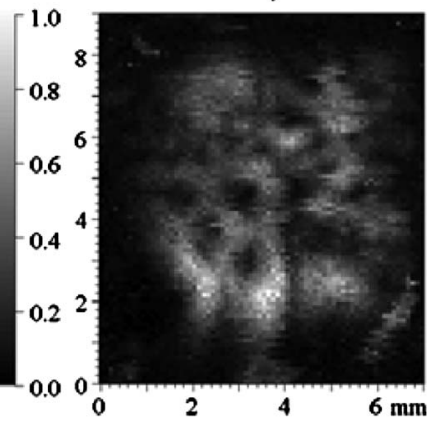

d)

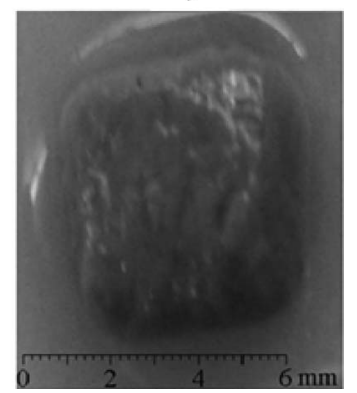

Fig. 4 Photoacoustic images of a piece of swine liver embedded in the chicken breast (a) before the HIFU ablation and (b) after HIFU ablation. The ultrasound intensity at the focal zone is $10^{3} \mathrm{~W} / \mathrm{cm}^{2}$, and the ablation duration is $4 \mathrm{~s}$. (c, d) are photographs of the swine liver before and after HIFU ablation, respectively.

standard deviation of 1.8. The increase in the amplitude of photoacoustic signals after HIFU treatment is consistent with previous results. ${ }^{16,17}$ The increase in the amplitude of photoacoustic signals after HIFU can be attributed to a combination of several factors. Notably, the Grüneisen parameter is affected by tissue coagulation. ${ }^{22}$ During this experiment, the ultrasound intensity at the focal region was estimated to be $10^{4} \mathrm{~W} / \mathrm{cm}^{2}$ during the HIFU ablation, and the treatment duration was $1 \mathrm{~s}$.

Photoacoustic images of the swine liver tissue embedded in chicken breast tissues before and after HIFU are presented in Fig. 4. Figure 4(a) was taken before HIFU ablation and shows the location of liver tissue and shape, which acted as the guidance for the subsequent HIFU ablation. For HIFU ablation, we chose the scanning area of $5.4 \times 7 \mathrm{~mm}$, which accurately covered the entire area of the liver and avoided burning the surrounding tissue. The step size of the scanning on the sample was $0.2 \mathrm{~mm}$. At each scanning position, the ultrasonic wave was focused into the sample for $4 \mathrm{~s}$, with a focal intensity of $10^{3} \mathrm{~W} / \mathrm{cm}^{2}$. Compared to Fig. 4(a), Fig. 4(b) (which is the photoacoustic image after HIFU ablation) reveals the swine liver tissue with approximately the same size and slight increase at PAI contrast, which is shown by the increased photoacoustic signal amplitudes in the MAP image. Figures 4(c) and 4(d) shows the photograph of the liver tissue before and after HIFU ablation.

To quantify the change in the photoacoustic image after HIFU, we calculated averaged enhancement in the amplitude of photoacoustic signals before and after HIFU in the HIFUtreated region. The average enhancement is $\sim 6 \%$ with a standard deviation of $4 \%$. The enhancement in photoacoustic sig- 
nals is small because the ultrasound intensity at the focal region was only $10^{3} \mathrm{~W} / \mathrm{cm}^{2}$, and the treatment duration was $4 \mathrm{~s}$ for current HIFU ablation. We anticipate that a higher enhancement in photoacoustic signal should be achieved if a higher ultrasound intensity level at the focal region is used during HIFU ablation, as shown in Fig. 3.

The results in Fig. 4 demonstrate that the combined PAI/ HIFU system with one transducer is capable of performing PAI-guided HIFU ablation. PAI can be performed to identify the location of the targeted region, and HIFU can then be performed to ablate the targeted tissue. At the end, PAI can be performed again to confirm the HIFU ablation.

\section{Discussion and Conclusions}

An integrated PAI and HIFU system was presented in current research. We demonstrated that with one system, PAI could be used as a guidance-and-evaluation tool for HIFU ablation. A combined reflection-mode PAI and HIFU with one transducer only needs one tissue window for both imaging and therapy, and therefore, it will be convenient to use in future clinic practice. With the combined system, precise alignments between treated areas and planned treatment areas, which are indentified on the image, can be assured relatively easy. As a result, the ablation during the therapeutic phase can be performed precisely on the area identified through the obtained image.

We used a 5-MHz HIFU transducer for PAI in this system. Usually, a focused, broadband ( $>70 \%$ bandwidth) transducer is preferred for the PAM. A broadband transducer will provide good axial resolution, whereas the lateral resolution is determined by the focusing effect of the transducer. For HIFU application, we usually use a narrowband transducer to achieve the best energy delivery. When a narrowband transducer is used for PAI, the axial resolution will become worse than that of using a broadband transducer, whereas lateral resolution should be the same because it depends on the focusing effect. In summary, when we use a HIFU transducer for PAI, we may sacrifice the axial resolution. When we use our HIFU transducer, which has a factional bandwidth of $\sim 50 \%$, as a PAI receiver, a reasonable axial resolution is obtained (Fig. 2). Compared to the benefits of the combined device, this axial resolution is acceptable. Therefore, we believe the proposed technique is feasible and promising.

Additionally, current system used a single-element transducer for PAI and HIFU; therefore, mechanical scans were required to conduct the imaging process and HIFU ablation. A phased array transducer can be used in the future to reduce the time required for imaging and therapy. We envision that the PAI technique may potentially be combined with HIFU ablation for image-guided therapy.

\section{Acknowledgment}

This work was supported in part by New Faculty General Research Fund at the University of Kansas

\section{References}

1. J. E. Kennedy, "High-intensity focused ultrasound in the treatment of solid tumours," Nat. Rev. Cancer 5(4), 321-327 (2005).

2. J. C. Rewcastle, "High intensity focused ultrasound for prostate cancer: A review of the scientific foundation, technology and clinical outcomes," Technol. Cancer Res. Treat. 5(6), 619-625 (2006).

3. G. Ter Haar, D. Sinnett, and I. Rivens, "High-intensity focused ultrasound-a surgical technique for the treatment of discrete livertumors," Phys. Med. Biol. 34(11), 1743-1750 (1989).

4. C. Damianou, K. Ioannides, V. Hadjisavvas, N. Mylonas, A. Couppis, and D. Iosif, "In vitro and in vivo brain ablation created by highintensity focused ultrasound and monitored by MRI," IEEE Trans. Ultrason. Ferroelectr. Freq. Control 56(6), 1189-1198 (2009).

5. K. Hynynen, O. Pomeroy, D. N. Smith, P. E. Huber, N. J. McDannold, J. Kettenbach, J. Baum, S. Singer, and F. A. Jolesz, "MR imaging-guided focused ultrasound surgery of fibroadenomas in the breast: a feasibility study," Radiology 219(1), 176-185 (2001).

6. C. M. C. Tempany, E. A. Stewart, N. McDannold, B. J. Quade, F. A. Jolesz, and K. Hynynen, "MR imaging-guided focused ultrasound surgery of uterine leiomyomas: a feasibility study," Radiology 226(3), 897-905 (2003).

7. K. Hynynen, "MRI-guided focused ultrasound treatments," Ultrasonics 50(2), 221-229 (2010).

8. J. E. Kennedy, F. Wu, G. R. ter Haar, F. V. Gleeson, R. R. Phillips, M. R. Middleton, and D. Cranston, "High-intensity focused ultrasound for the treatment of liver tumours," Ultrasonics 42(1-9), 931-935 (2004).

9. L. V. Wang, "Prospects of photoacoustic tomography," Med. Phys. 35(12), 5758-5767 (2008).

10. R. A. Kruger, P. Y. Liu, Y. R. Fang, and C. R. Appledorn, "Photoacoustic ultrasound (paus)-reconstruction tomography," Med. Phys. 22(10), 1605-1609 (1995).

11. C. G. A. Hoelen, F. F. M. de Mul, R. Pongers, and A. Dekker, "Threedimensional photoacoustic imaging of blood vessels in tissue," Opt. Lett. 23(8), 648-650 (1998).

12. R. O. Esenaliev, A. A. Karabutov, and A. A. Oraevsky, "Sensitivity of laser optoacoustic imaging in detection of small deeply embedded tumors," IEEE J. Sel. Top. Quantum Electron. 5(4), 981-988 (1999).

13. R. A. Kruger, D. R. Reinecke, and G. A. Kruger, "Thermoacoustic computed tomography-technical considerations," Med. Phys. 26(9), 1832-1837 (1999).

14. M. H. Xu and L. H. V. Wang, "Photoacoustic imaging in biomedicine," Rev. Sci. Instrum. 77(4), 041101 (2006)

15. G. Ku and L. H. V. Wang, "Deeply penetrating photoacoustic tomography in biological tissues enhanced with an optical contrast agent," Opt. Lett. 30(5), 507-509 (2005).

16. T. D. Khokhlova, I. M. Pelivanov, O. A. Sapozhnikov, V. S. Solomatin, and A. A. Karabutov, "Opto-acoustic diagnostics of the thermal action of high-intensity focused ultrasound on biological tissues: the possibility of its applications and model experiments," Quantum Electron. 36(12), 1097-1102 (2006).

17. K. V. Larin, I. V. Larina, and R. O. Esenaliev, "Monitoring of tissue coagulation during thermotherapy using optoacoustic technique," $J$. Phys. D 38(15), 2645-2653 (2005).

18. A. R. Funke, J. F. Aubry, M. Fink, A. C. Boccara, and E. Bossy, "Photoacoustic guidance of high intensity focused ultrasound with selective optical contrasts and time-reversal," Appl. Phys. Lett. 94(5), 054102 (2009).

19. K. H. Song and L. V. Wang, "Deep reflection-mode photoacoustic imaging of biological tissue," J. Biomed. Opt. 12(6), 060503 (2007).

20. K. Maslov, G. Stoica, and L. H. V. Wang, "In vivo dark-field reflection-mode photoacoustic microscopy," Opt. Lett. 30(6), 625627 (2005).

21. W. F. Cheong, S. A. Prahl, and A. J. Welch, "A review of the opticalproperties of biological tissues," IEEE J. Quantum Electron. 26(12), 2166-2185 (1990).

22. B. Soroushian, W. M. Whelan, and M. C. Kolios, "Assessment of opto-mechanical behavior of biological samples by interferometry," Proc. SPIE 7177, 71771X (2009). 